LAWRENCE LIVERMORE N A T IO N A L LABORATORY

\title{
Atomic Control Of Water Interaction With Biocompatible Surfaces: The Case Of $\mathrm{SiC}(001)$
}

G. Cicero, A. Catellani, G. Galli

July 28,2004

Atomic Control Of Water Interaction With Biocompatible Surfaces? The Case Of $\mathrm{SiC}(001)$ 
This document was prepared as an account of work sponsored by an agency of the United States Government. Neither the United States Government nor the University of California nor any of their employees, makes any warranty, express or implied, or assumes any legal liability or responsibility for the accuracy, completeness, or usefulness of any information, apparatus, product, or process disclosed, or represents that its use would not infringe privately owned rights. Reference herein to any specific commercial product, process, or service by trade name, trademark, manufacturer, or otherwise, does not necessarily constitute or imply its endorsement, recommendation, or favoring by the United States Government or the University of California. The views and opinions of authors expressed herein do not necessarily state or reflect those of the United States Government or the University of California, and shall not be used for advertising or product endorsement purposes. 


\title{
Atomic control of water interaction with bio-compatible surfaces: the case of $\mathrm{SiC}(001)$
}

\author{
Giancarlo Cicero \\ INFM \& Physics Dept., Torino Polytechnic, \\ C.Duca degli Abruzzi, 24, I-10129 Torino, Italy* \\ Alessandra Catellani \\ CNR-IMEM, Parco Area delle Scienze, 37A, I-43010 Parma, Italy \\ Giulia Galli \\ Lawrence Livermore National Laboratory, \\ P.O. Box 808, Livermore CA94550 USA
}

(Dated: July 23, 2004)

\begin{abstract}
The interaction of water with $\mathrm{Si}$ - and $\mathrm{C}$ - terminated $\beta$-SiC(001) surfaces was investigated by means of $a b$ - initio molecular dynamics simulations. Irrespective of coverage, varied from $1 / 4$ to 1 monolayer, we found that water dissociates on the Si-terminated surface, substantially modifying the clean surface reconstruction, while the C-terminated surface is non reactive and hydrophobic. Based on our results, we propose that STM images and photoemission experiments may detect specific changes induced by water on both the structural and electronic properties of $\mathrm{SiC}(001)$ surfaces.
\end{abstract}

PACS numbers: 68.08.De, 68.43.-h 
The interaction of water with surfaces plays a central role in a number of fields, ranging from biophysics to semiconductor technology and corrosive processes. In particular, the characterization of the interface of water with biocompatible solids is key for designing devices operating in a biological environment, e.g. for patterning surfaces at the molecular level and then using these patterns to control adsorption of proteins while maintaining their activity [1-3].

The search for biocompatible, patterned substrates for the development of bio-sensors is an active field of research [4,5]. Silicon Carbide is considered to be a very promising material for biophysics applications, due to its low weight, high strength, extreme hardness, wear and corrosion resistance, and inertness. However, studies of water-SiC interactions are still in their infancy [6], since the microscopic characterization of $\mathrm{H}_{2} \mathrm{O} / \mathrm{SiC}$ interfaces is extremely challenging, both from an experimental and a theoretical standpoint. Only very recently a robust experimental characterization of clean $\mathrm{SiC}$ surfaces has been obtained, with unexpected results [7]. Theoretically, an investigation of water on $\mathrm{SiC}$ calls for the use of refined quantum mechanical methods, which are very demanding for the complex geometrical and electronic configurations such as those involved in the study of $\mathrm{H}_{2} \mathrm{O}$ molecules on a polar surface.

First-principles methods have proven to be very useful to elucidate the mechanisms of water adsorption on both ionic [8] and semiconductor surfaces [9-12]. Recently it has been established that at room temperature water is preferentially adsorbed on Si via a dissociative chemisorption process [9-11]. In contrast, on diamond (001) surfaces [12, 13] dissociation is unlikely to occur because of a high activation energy barrier, although it is energetically favored. For highly ionic compounds, calculations have shown that reactivity with water strongly depends on both the local environment and the charge transfer occurring in the uppermost atomic layers. $\mathrm{SiC}$ is a wide-band gap semiconductor with partially ionic bonds and the $\mathrm{Si}$ and $\mathrm{C}$ terminated surfaces of the cubic polytype have structural and elastic properties very different from their silicon and diamond counterparts. Therefore SiC surfaces represent a particularly complex case when it comes to unravelling the interaction with water.

Here we report on predictions of water interaction with $\mathrm{SiC}$ substrates obtained using $a b$-initio molecular dynamic simulations. We considered both Si- and C-terminated surfaces respectively in their stable $\mathrm{p}(2 \times 1)$ and $\mathrm{c}(2 \times 2)$ surface reconstructions [14] and we investigated different molecular coverages. Irrespective of the coverage, we found that water 


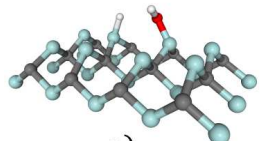

a)

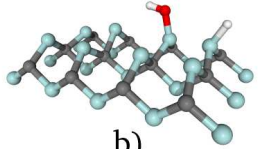

b)

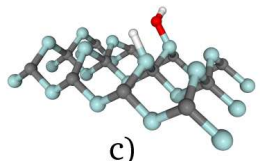

c)

FIG. 1: Dissociated water configurations on the Si-terminated $\operatorname{SiC}(001)$ surface. Schematic side view of dissociation geometries of one water molecule on $\mathrm{Si}-\mathrm{SiC}(001)$ : grey (blue) spheres represent C (Si) atoms, while red (white) spheres represent $\mathrm{O}(\mathrm{H})$ atoms. Only the three outermost slab layers are indicated.

molecules dissociate on the Si-terminated [Si-SiC(001)] surface and that at high coverage full hydroxylation occurs via a proton exchange mechanism similar to that found on alumina [8]. On the other hand, water dissociation is found to be an activated process on the Cterminated [C-SiC(001)] surface, and it is very unlikely to occur even at high temperature. Our findings suggest that by preparing SiC substrates with adjacent C- and Si-terminated islands, nanoscale hydrophobic/hydrophilic patterns can be created, which could be suitable for functionalization with different types of biomolecules. Our results also permit to identify specific changes induced on the Si-terminated surface by water chemisorption, as a function of coverage, and we suggest that these could be evidenced by Scanning Tunnelling Microscopy (STM) experiments.

Our calculations have been performed within Density Functional Theory in the Generalized Gradient corrected Functional (PBE), as parameterized by Perdew et al. [15]. In our simulations, the $\mathrm{SiC}(001)$ surfaces were represented by a symmetric slab, periodically repeated along the (001) direction [16]. The geometry of all configurations was optimized using ab-initio molecular dynamics $[17,18]$ at the theoretical equilibrium lattice parameter. We used nonlocal pseudopotentials [19] derived within the PBE approximation. The electronic wavefunctions (charge density) were expanded in plane waves, with energy cutoff up to 80 (320) Ry. Similar to previous investigations [14], the integration over the Brillouin Zone was performed using only the $\Gamma$ point [16].

We first investigated the reaction of water molecules with the $\mathrm{Si}-\mathrm{SiC}(001)$ surface at $\sim$ 1/4 ML coverage [20] (i.e. one water molecule for each surface dimer). The surface/molecule interaction is not negligible even at large distances: a water molecule at $5.8 \AA$ from the surface already induces a considerable shortening of the Si-dimers. At nearly zero temperature, as the distance to the $\mathrm{Si}$ surface atoms is decreased, $\mathrm{H}_{2} \mathrm{O}$ molecules spontaneously dissoci- 
ate. Irrespective of its physisorption position, a $\mathrm{H}_{2} \mathrm{O}$ molecule diffuses on the surface until it finds a favorable dissociation site, and then it spontaneously breaks into two fragments $(-\mathrm{H}$ and $-\mathrm{OH})$ bonded to two adjacent Si atoms after a short simulation time $(\sim 0.5-1.0 \mathrm{ps}$, depending on the starting position and orientation). We observed three possible dissociation configurations which differ by the relative position of the $-\mathrm{H}$ and $-\mathrm{OH}$ groups (see Fig. 1), though being very similar in the local geometry. In particular, a Si-O bond (1.66 $\AA$ ) and a Si-H bond (1.49 $\AA$ ) are formed, and the surface Si dimers are shortened with respect to those on the clean surface (from 2.64 to $2.46 \AA$ ), acquiring a buckling $(\Delta \mathrm{z})$ of about $0.1 \AA$. The energy gain upon dissociation is very similar, being 3.3, 3.2 and $3.5 \mathrm{eV} / \mathrm{molec}(77.2$, 74.3 and $80.2 \mathrm{Kcal} / \mathrm{mol}$ ) for the three cases a, b, and c of Fig. 1, respectively.

Water interaction with the $\mathrm{Si}-\mathrm{SiC}$ surface gives rise to chemisorbed states qualitatively similar to those observed on the $\operatorname{Si}(001)$ surface, where a water molecule was found to dissociate after forming a transient molecular physisorbed state [9-11]. However the dissociation mechanism and the final products do differ from those of $\operatorname{Si}(001)$, mainly because of the different bonding properties and hence reconstruction of the clean $\mathrm{Si}-\mathrm{SiC}(001)$ and $\mathrm{Si}(001)$. Indeed the silicon clean surface presents buckled dimers which elongate when interacting with a water molecule, reducing their tilt to zero as the molecule dissociates [9-11]. Here the opposite occurs: the $\mathrm{Si}-\mathrm{SiC}(001)$ flat dimers are shortened and become buckled upon water dissociation. Furthermore, in the case of the Si surface, the product with the $-\mathrm{H}$ and -OH fragments bound to the same surface dimer was found to be the most stable, while on the $\mathrm{Si}-\mathrm{SiC}$ surface this is the most unfavorable configuration (case b of Fig. 1).

At this low water coverage we observed distortions of the original surface reconstruction induced by the presence of $-\mathrm{OH}$ groups. For example, for configuration b) in Fig. 1, we observed that the hydrogen atom of the -OH fragment directly points to the upper Si atom of the adjacent Si-Si dimer, which turns out to be electron rich at variance with its geometric configuration on the clean surface. A hydrogen-like bond (-OH $\cdots \mathrm{Si}$ ) is then formed, characterized by an $\mathrm{O} \cdots$ Si distance of $3.66 \AA$ ( $\mathrm{H} \cdots \mathrm{Si}=2.94 \AA)$. These structural modifications are accompanied by changes in the electronic structure of the surface which could be detected in STM experiments. Fig. 2 (left panel) shows a theoretical STM image of the Si-SiC(001) surface with a $1 / 4$ water monolayer, obtained using the local density of state method [21]. We find that $-\mathrm{OH}$ and $-\mathrm{H}$ groups are dark, while bright spots are found in correspondence of upper Si atoms of the surface dimers, which in the absence of water would be basically 
flat.

We note that the structural changes observed on Si-SiC(001) upon water adsorption and in particular the transition from almost flat, long Si-Si dimers to buckled, short dimers - can explain the long-standing controversy about structural data obtained for $\mathrm{SiC}(001)$ by different groups [14]. Data taken in different vacuum conditions may have recorded different structures, since even minute quantities of water (or air) induce substantial changes to the surface geometry.

In order to understand if on $\mathrm{Si}-\mathrm{SiC}(001)$ molecular dissociation occurs as well in a "liquidlike" environment, where hydrogen bonds among molecules are present, we first considered the reactivity of a water dimer with this surface. We observed the same reaction path towards dissociation found for a single molecule and the same dissociation energy gain. We then simulated a high coverage adsorption depositing one randomly oriented layer of water molecules on a $\mathrm{Si}-\mathrm{SiC}$ surface, i.e. 4 molecules for each $\mathrm{c}(4 \times 4)$ surface. This corresponds to a monolayer at the density of liquid water at room $\mathrm{T}\left(1 \mathrm{~g} / \mathrm{cm}^{3}\right)$. At high coverage, we found that the dissociation process mainly occurs through a proton exchange mechanism among the water molecules. In some cases a synchronous two proton transfer occurs (see Fig. 3), which involves two neighboring molecules connected by a hydrogen bond and a surface Si atom: In Fig. 3, molecule 1 is physisorbed to one Si atom and makes a hydrogen bond with molecule 2 ; the proton involved in the $\mathrm{H}$-bond is transferred from molecule 1 to molecule 2, which releases a hydrogen atom in order to remain neutral. This $\mathrm{H}$ atom is eventually bonded to a nearby $\mathrm{Si}$ atom. It is interesting to note that at variance with the dissociation of a water molecule observed at low coverage, in this case the $-\mathrm{OH}$ and $-\mathrm{H}$ fragments are not necessarily bonded to two first neighboring Si atoms. A proton transfer mechanism similar to the one observed here has been found in an ab-initio study of water molecules on alumina [8] which, similar to $\mathrm{SiC}$, presents polar surfaces. However in the case of $\mathrm{Al}_{2} \mathrm{O}_{3}$, a temperature of $300 \mathrm{~K}$ was required to observe the process, while in this case it occurs unexpectedly at very low temperature. Our total energy calculations show that full hydroxylation of the $\mathrm{Si}-\mathrm{SiC}(001)$ surface is favored. The dissociation energy per molecule $(3.2 \mathrm{eV} / \mathrm{molec}-73.6 \mathrm{Kcal} / \mathrm{mol})$ is only slightly lower than the one obtained at low coverage. The results of our calculations at full coverage are in qualitative agreement with those of recent experiments on water adsorbed on the Si-rich (3x2)-reconstructed $\mathrm{SiC}(001)$ surface [6] where complete dissociation of water molecules leading to perfect passivation of 

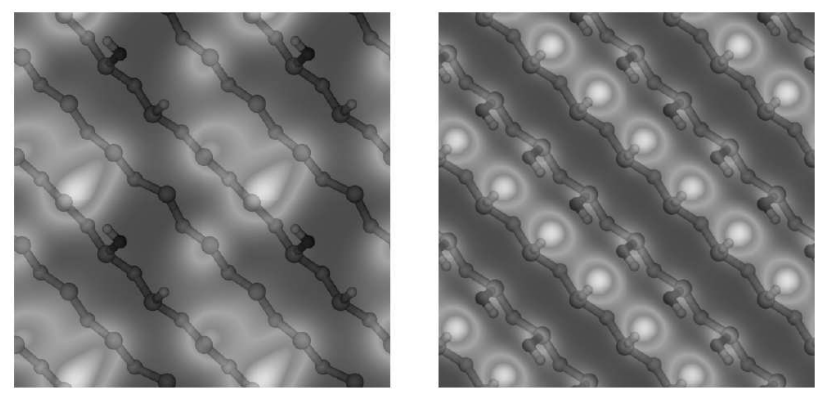

FIG. 2: Computed filled state Scanning Tunnelling Microscopy (STM) images of the Si-terminated $\mathrm{SiC}(001)$ surface after water exposure. The most favorable 1/4 (left) and 1ML (right) configurations for water on $\mathrm{Si}-\mathrm{SiC}(001)$ surface as obtained with a tip voltage of $-1.5 \mathrm{~V}$. The geometry of the last two surface layers and that of the dissociated $-\mathrm{H}$ and $-\mathrm{OH}$ fragments are projected on the image to identify the surface geometric structure.

the outermost excess Si layer was observed.

The high density of -OH groups formed at the Si-terminated surface upon water adsorption is expected to enhance the surface hydrophilic character. Indeed the Si-OH group can form hydrogen bonds and then interact efficiently with water [22].

Similar to the low coverage case, structural changes upon water chemisorption are accompanied by electronic structure changes, which can be seen in STM images. However the states shown in theoretical STM pictures at high coverage (Fig. 2, right panel) are very different from those seen at low coverage. In particular, we find that in this case the brightest spots do not correspond to electronic states localized on Si atoms but to states localized across the dimer rows and pointing from the hydrogen to the -OH groups. These results provide a microscopic model of images detected in STM experiments, which may be used to reveal water adsorption on the surface, as a function of coverage. Furthermore we note that, due to the saturation of all the dangling bonds of the surface atoms upon water adsorption, at full coverage the optical gap increases giving rise to a wide band gap substrate (the gap computed with the approximations used in our calculation [23] is $1.6 \mathrm{eV}$ ). This increase may be observed in photoemission experiments.

A completely different reactivity with water molecules has been found on the Cterminated surface. Indeed, for every tested adsorption site [20], we found that $\mathrm{H}_{2} \mathrm{O}$ molecules leave the surface and, at the end of the simulation, remain floating well above the substrate, leaving the surface reconstruction nearly unperturbed. This behavior is consis- 
tent with the calculated total energy difference between the weakly interacting surface $/ \mathrm{H}_{2} \mathrm{O}$ system and the non interacting constituents, which turned out to be essentially zero. The same qualitative results have also been obtained at high coverage (1 ML). In this case the water molecules move to optimize the hydrogen bond network, without interacting with the underlying surface atoms over a short $(\sim 1.3 \mathrm{ps})$ simulation run. However, when a water molecule is pushed towards a surface carbon atom at a distance lower than $1.5 \AA$, thus overcoming a rather large energy barrier, it quickly dissociates. The $-\mathrm{OH}$ and $-\mathrm{H}$ fragments bind to the two different $\mathrm{C}$ atoms of the surface dimer, with an energy gain of $1.4 \mathrm{eV} / \mathrm{molec}$ $(31.6 \mathrm{Kcal} / \mathrm{mol})$. Upon dissociation, the C-dimer which reacted with water has an elongated bond $\left(\sim 1.36 \AA\right.$ ) with $\mathrm{sp}^{2}$ hybridized atoms; in addition the dimer acquires a buckling of $2.4^{\circ}$ ( $\Delta \mathrm{z}=0.03 \AA$ ), where the $\mathrm{C}$ atom bonded to the oxygen atom is in the upper position (C-O $1.40 \AA$ ). The energy barrier for this reaction has been estimated [24] to be of the order of $1.0 \mathrm{eV} / \mathrm{molec}(23.1 \mathrm{Kcal} / \mathrm{mol})$, which shows that even at high $\mathrm{T}$ the water/surface reaction does not spontaneously occur. This behavior qualitatively resembles the reactivity of the (001) surface of diamond. As shown both theoretically [12] and experimentally [13], water does not form any molecular precursor state on diamond and dissociation is an extremely exothermic $(2.7 \mathrm{eV} / \mathrm{molec}-63.0 \mathrm{Kcal} / \mathrm{mol})$ and activated process (the activation barrier is $\sim 1.1 \mathrm{eV} / \mathrm{molec}-25.8 \mathrm{Kcal} / \mathrm{mol})$. However, while the diamond surface is left with single C-C bonds upon water dissociation, the C-SiC(001) surface still presents strongly bonded $\mathrm{sp}^{2}$ atoms.

These results show that at variance with the $\mathrm{Si}-\mathrm{SiC}(001)$, the carbon terminated surface is highly inert towards a wet ambient, resulting in a hydrophobic environment. This suggests that by growing $\mathrm{SiC}$ substrates with adjacent islands which are either Si- or C-terminated an atomic control of hydrophilic and hydrophobic environments may be attained. This could be realized by epitaxial growth of cubic $\mathrm{SiC}$ substrates which are preferentially Siterminated and by subsequent deposition of, e.g. $\mathrm{C}_{2} \mathrm{H}_{4}$ groups on masked islands, along the lines proposed in Ref. [25]. Controlling wetting properties of a surface by patterning regions with hydrophilic and hydrophobic characters is very important for the attachment of DNA and proteins and the realization of sensors $[1,2,5]$. Finally the use of SiC-based biosensors would open the way to applications where the requirement of biocompatibilty is crucial, i.e selective bio-organism capture [26] and drug testing.

Use of the computing facilities at CINECA (I) (INFM Parallel Computing Initiative) 


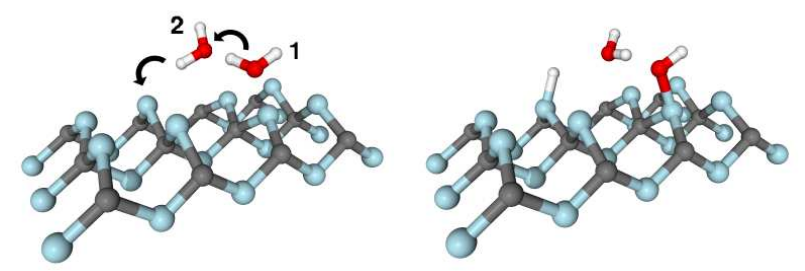

FIG. 3: Dissociation mechanism of water on the Si-terminated $\mathrm{SiC}(001)$ surface at high coverage. Two successive snapshots of the dissociation process at 1ML water coverage on Si-SiC(001). Only the two molecules involved in proton exchange during this time interval are represented and labelled as 1 and 2. Colors as in Fig. 1. Only the three outermost slab layers are indicated.

and at the University of California, Lawrence Livermore National Laboratory (LLNL) is gratefully acknowledged. Part of this work was performed under the auspices of the U.S. Department of Energy by University of California Lawrence Livermore National Laboratory under contract No. W-7405-Eng-48. One of us (G.C.) is grateful to the CCMS Summer Institute and LLNL for hospitality and financial support. We also wish to thank D. Gerion and J. Grossman for fruitful scientific discussions.

* Now at LLNL, CA-USA; Electronic address: giancarlo.cicero@polito.it

[1] J. T. C. Wojtyk, M. Tomietto, R. Boukherroub, and D. D. M. Wayner, J. Am. Chem. Soc. 123, 1535 (2001).

[2] S. D. Gillmor, A. J. Thiel, T. C. Strother, L. M. Smith, and M. G. Lagally, Langmuir 16, $7223(2000)$.

[3] J. M. Buriak, Chem. Rev. 102, 1271 (2002).

[4] L. Tiefenauer and R. Ros, Colloids and Surfaces B: Biointerfaces 23, 95 (2002).

[5] A. P. Blanchard, R. J. Kaiser, and L. E. Hood, Biosens. Bioelectrons. 11, 687 (1996).

[6] F. Amy and Y. J. Chabal, J. Chem. Phys. 119, 6201 (2003).

[7] V. Derycke, P. G. Soukiassian, F. Amy, Y. J. Chabal, and H. B. Enriquez, Nature Mater. 2, $253(2003)$.

[8] K. C. Hass, W. F. Schneider, A. Curioni, and W. Andreoni, Science 282, 265 (1998).

[9] J. Cho, K. S. Kim, S. Lee, and M. Kang, Phys. Rev. B 61, 4503 (2000). 
[10] R. Konecny and D. J. Doren, J. Chem. Phys. 106, 2426 (1997).

[11] Y. Okamoto, Phys. Rev. B 60, 10632 (1999).

[12] Y. Okamoto, Phys. Rev. B 58, 6760 (1998).

[13] L. M. Struck and M. P. D’Evelyn, J. Vac. Sci. and Technol. A 11, 1992 (1993).

[14] A. Catellani and G. Galli, Prog. Surf. Sci. 69, 101 (2002).

[15] K. B. J. P. Perdew and M. Ernzerhof, Phys. Rev. Lett. 77, 3865 (1996).

[16] We used supercells with 11 layers, 8 atoms/layer and a $\sim 12.5 \AA$ vacuum region, between clean surfaces. Some calculations were performed on a 11 layers, 16 atoms/layer slab, to check size effects and allow for a more disordered distribution of molecules. The $\Gamma$ point corresponds to 3 (6) inequivalent k-points in the primitive cell for the supercell with 8 (16) atoms/layer. Atomic configurations were considered converged when atomic forces were less than $10^{-4}$ a.u./atom and the energy varied by less than $310^{-5} \mathrm{eV} /$ atom.

[17] R. Car and M. Parrinello, Phys. Rev. Lett. 55, 2471 (1985).

[18] We used the first principles molecular dynamics code gp (F. Gygi, LLNL, 1998-2003).

[19] D. Hamann, Phys. Rev. B 40, 2980 (1989).

[20] We considered different orientations of the plane defined by a water molecule (parallel or perpendicular to the surface), and we considered the molecule impinging either with oxygen or hydrogen atoms.

[21] J. Tersoff and D. R. Hamman, Phys. Rev. B 31, 805 (1985).

[22] We have estimated that the $\mathrm{H}$ bond between this group and a water molecule is $0.17 \mathrm{eV} / \mathrm{bond}$ $(3.8 \mathrm{Kcal} /$ bond $)$ or $0.27 \mathrm{eV} /$ bond $(6.3 \mathrm{Kcal} /$ bond $)$, when it acts as proton acceptor or donor respectively. Note that the hydrogen bond between two water molecules is about $0.20 \mathrm{eV} / \mathrm{bond}$ - $4.7 \mathrm{Kcal} /$ bond. Those values have been obtained from calculations on molecular systems: hydrogen bonded silanol/water and water/water molecules.

[23] This is expected to be an underestimate of the experimental value: LDA is known to underestimate optical gaps in sp-bonded solids.

[24] We performed a 10 step constrained molecular dynamics starting with a water molecule facing C-dimers, with an $\mathrm{OH}$ bond parallel to the surface with increasing $\mathrm{O}-\mathrm{H}$ distance $\left(\mathrm{d}_{\mathrm{OH}}\right)$. At each step, all the atoms were allowed to relax, while keeping constant $d_{\mathrm{OH}}$. The energy path has a maximum when $\mathrm{d}_{\mathrm{OH}}$ is $1.25 \AA$; the estimated energy barrier along this path is $\sim 1.4$ $\mathrm{eV} / \mathrm{mol}$. 
[25] J. P. Long, V. M. Bermudez, and D. E. Ramaker, Phys. Rev. Lett. 76, 991 (1996).

[26] S. E. Lètant, B. R. Hart, A. W. V. Buuren, and L. J. Terminello, Nature Mater. 2, 391 (2003). 